\title{
A Survey on Compressive Sensing Techniques for Cognitive Radio
}

\section{Networks}

1

2 Fatima Salahdine ${ }^{1,2}$. Naima Kaabouch ${ }^{1}$. Hassan El Ghazi ${ }^{2}$

3

4

5

6

Received: date / Accepted: date Nyquist theorem, channel estimation, MIMO.

\section{Introduction}

Abstract- In cognitive radio, one of the main challenges is wideband spectrum sensing. Existing spectrum sensing techniques are based on a set of observations sampled by an analog/digital converter (ADC) at the Nyquist rate. However, those techniques can sense only one band at a time because of the hardware limitations on sampling rate. In addition, in order to sense a wideband spectrum, the band is divided into narrow bands or multiple frequency bands. Secondary users (SU) have to sense each band using multiple RF frontends simultaneously, which results in a very high processing time, hardware cost, and computational complexity. In order to overcome this problem, the signal sampling should be as fast as possible, even with high dimensional signals. Compressive sensing has been proposed as one of the solutions to reduce the processing time and accelerate the scanning process. It allows reducing the number of samples required for high dimensional signal acquisition while keeping the important information. Over the last decade, a number of papers related to compressive sensing techniques have been published. However, most of these papers describe techniques corresponding to one process either sparse representation, sensing matrix, or recovery. This paper provides an in depth survey on compressive sensing techniques and classifies these techniques according to which process they target, namely, sparse representation, sensing matrix, or recovery algorithms.It also discusses examples of potential applicationsof these techniques including in spectrum sensing, channel estimation, and multiple-input multiple-output (MIMO) based cognitive radio.Metrics to evaluate the efficiencies of existing compressive sensing techniques are providedas well as the benefits and challenges in the context of cognitive radio networks.

KeywordsCognitive radio network, spectrum sensing, compressive sensing, sparsity representation, sensing matrix, processing time, recovery algorithms, restrict isometry property, analog to digital converter, Shannon-

Over the last decade, a number of spectrum sensing techniques have been proposed to detect and locate dynamically unused spectrum channels in a band of interest [1-6]. Examples of these techniques include energy detection [7], matched filter [8], autocorrelation [9-11], and wavelet based detection [12].Energy detection operates by comparing the SU signal average energy with an estimated threshold. This technique does not

Fatima Salahdine

e-mail: salahdine@und.edu

Naima Kaabouch

e-mail: naima.kaabouch@engr.und.edu

Hassan El Ghazi

e-mail: $\underline{\text { elghazi@inpt.ac.ma }}$

${ }^{1}$ Electrical Engineering Department, University of North Dakota, Grand Forks, USA

${ }^{2}$ STRS Lab, National Institute of Posts and Telecommunication, Rabat, Morocco 
require any knowledge of the PU signal and itis simple and easy to implement. However,it has high false detection ratesand it is not able to distinguish between signals and noise [7]. Matched filter requires the knowledge of the PU signal characteristics including frequency, modulation type, and bandwidth. It operates by comparing the matched filter output with a threshold [8].Autocorrelation based detection requires the knowledge of the statistical distribution of the autocorrelation function. It operates by comparing the autocorrelation function at lag 0 with that at lag 1.This technique is able to differentiate between signals and noise, which makes it less sensible to noise uncertainty [9-12]. Waveletbased sensing is based on wavelet transform to detect the singularities of the power spectral density (PSD). It works by computing the wavelet transform of the SU signal and calculating PSD, which is then compared to a threshold to make the decision about the signal presence. This technique does not require the knowledge of the PU signal;however, it requires the mother wavelet to be chosen appropriately in order to operateefficiently[13].

40 Theabove sensing techniques, also called non-compressive sensing detectors, are based on a set of observations sampled by an analog/digital converter ADC at the Nyquist rate [14]. However, these techniques can sense only one band at a time because of the hardware limitations on sampling rate. In addition, in order to sense a wideband spectrum, the band is divided into narrow bands or multiple frequency channels. The SU has to sense each band using multiple RF frontends simultaneously, which can result in a very high processing time, hardware cost, and computational complexity. To overcome some of these limitations, compressive sensing has been proposed as one of the solutions to reduce the processing time and accelerate the scanning process. It allows reducing the number of samples required for high dimensional signal acquisition while keeping the important information [15-17].

49 In compressive sensing theory, a signal can be acquired and compressed simultaneously in the same process with 50 only the essential information. The signal can be then recovered from few measurements at the Nyquist rate or 51 less. Compressive sensing can be achieved by respecting certain requirements, including sparsity, restrict 52 isometry property (RIP), and incoherence. This approach has been applied in several domains, in which it offered good results over the conventional approaches. Examples of these domains are radar systems [18], medical systems for rapid magnetic resonance imaging (IRM) [19, 20], and wireless sensor networks for signal acquisition [21]. In cognitive radio, this approach is also applicable because of the signal sparsity feature, which is valid in most of spectrum sensing scenarios.

57 Compressive sensing was also applied on channel estimation to overcome the limitations of the existing channel estimation techniques, known as non-compressive sensingchannel estimationtechniques. A number of these techniques have been proposed in the literature to evaluate and approximate the channel behavior [22-27]. Examples of these techniques include pilot-aided[22-24], blind [25], and least mean square (LMS) based channel estimation [26]. The channel estimation pilot aided techniques include a number of schemes such as minimum mean squared error (MMSE) and maximum likelihood estimator (MLE) based technique [22-24].In [23], a low rank estimation technique based on MMSE has been proposed for estimating channel pilots using singular value decomposition. This technique requires the SNR and channel frequency correlation to be known. In [24], the MLEwas used for channel estimation in the context of OFDM-MIMO. Unlike the MMSE based method, this technique does not require the knowledge of the channel information or SNR to operate.Theblind channel estimation technique consists on using the data symbols to estimate the parameters of the channel. This technique is not efficient for fading channels and represents high complexity [25]. LMS based channel estimation represents 
low complexity compared to the other techniques and it consists on computing the LMS based on the transmitted signal over the channel and the identity matrix [26].

The aforementioned techniques represent high complexity and often require the knowledge of the channel response at the receiver for multipath wireless channels, which is not always possible in practice because of the high number of required antennas[22-26].Moreover, multichannel signals are known to be sparse, which canlead to high estimation errors at the receiver. In order to overcome these limitations and exploit the sparsity of the multipath wireless channels, compressive sensing channel estimation techniqueshave been proposed and investigated, especially for MIMO-OFDM communication systems [27].

A number of papers and surveys related to compressive sensing have been published. However, most of these papers describe compressive sensing techniques corresponding to one process either sparse representation, sensing matrix, or recovery. Other papers focus on one of the compressive sensing category; and a few focus on the compressive sensing applications. Thus, there is a need for detailed review papers that compare and analyze the current compressive sensing techniques. Therefore, this paper provides a detailed overviewon compressive sensing techniquesand classifies these techniques according to which process they target, namely, sparse representation, sensing matrix, or recovery algorithms. The paper also discusses each category, some techniques under each category, and provides a comparison of these categories. In addition, examples of compressive sensing applications in cognitive radio networks, MIMO, and channel estimation, are discussed.

In this paper, we present a detailed overview of compressive sensing techniques and their applications in cognitive radio systems. The rest of this paper is organized as follows. Section II presents the compressive sensing model and its requirements. Section III reviews the sensing techniques classification, which is based on the involved process. Section IV presents compressive sensing potentialapplications in conventional and MIMO cognitive radio contexts. Subsequently, it also discusses compressive sensing limitations and tradeoffs involved in selecting which compressive sensing scheme to adopt. Finally, a conclusion is given at the end.

\section{Compressive sensing theory}

The concept of compressive sensing is introduced by Candes as a new approach to sample signals at or below the Nyquist rate $[28,29]$. Traditional approaches were based on Shannon-Nyquist theorem, in which it is possible to recover a signal at the receiver only if it is sampled at the Nyquist rate.

Compressive sensing mechanism is an acquisition process followed by a reconstruction algorithm. It combines sampling and sensing processes in one. Fig. 1 illustrates the three main stages of compressive sensing, which are sparse representation, encoding, and decoding. In the sparse representation stage [30], a sparse representation of the signal is found over a basis that permits to reconstruct the signal as accurately as possible. In the next stage, the sparse signal is sampled and compressed based on a measurements matrix [31-33]. In the last stage, the compressed signal is then reconstructed using a reconstruction algorithm [15, 29, 34, 35].

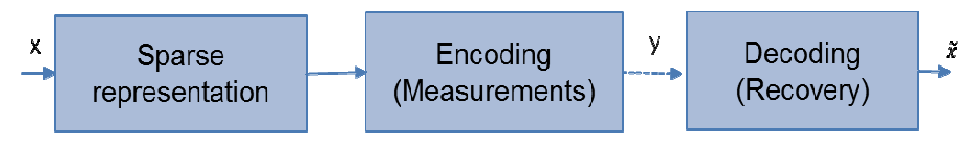

Fig. 1.Compressive sensing model.

Considering a high dimensional signal $x$ with a huge number of samples $N, x$ is assumed to be sparse in some domain, and it is $k$-sparse signal with $k<<$. Sparse representation consists on representing the signal by a number 
of projections on a suitable sparse basis $\varphi$, also known as dictionary or projection. Examples of sparse basis include wavelet transform, Fourier transform, and discrete cosine transform. Non sparse signal can be represented as a sparse signal by some sparse transforms. Every sparse signal can be represented in a scarifying basis $\varphi$ as follows

$$
x=\varphi * S
$$

where $s$ is the signal's projection on the sparse basis $\varphi(\mathrm{N} * \mathrm{~N})$, and $\|s\|_{0}=k \ll N[36,37]$. The sparse signal $x$ is compressed using the sensing matrix $\Phi$. The compression consists on multiplying the signal with an $M * N$ matrix. $M$ is much smaller than $N$ and it represents the measurements number that includes the essential information of $x$. For efficient recovery, $M$ should respect this condition $M=\mathrm{O}(k \log (N))$. The compressed signal is given by

$$
y=\Phi * x(2)
$$

where $y(M, 1)$ denotes the signal measurements, which selects only $M$ samples from $x(N, 1), M<<N$. $\Phi$ is the sensing matrix $(M, N)$ as shown in Fig. 2

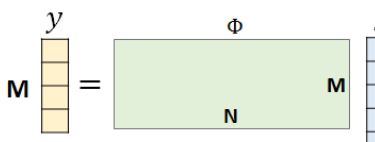

$\mathbf{N}$

Fig. 2. Compressive sensing structure.

From equations (1) and (2), compressed signal can be reformulated as

$$
y=\Phi * x=A * s(3)
$$

where $A=\Phi * \varphi$ is an $M * N$ matrix, known as recovery matrix, it satisfies the restricted isometry property. The last step consists in reconstructing the signal at the receiver, which implied solving the equation (3). It aims to solve an undetermined system with more unknowns than the number of equations. Because of the sparsity assumption, it is possible to estimate a high dimensional signal $N$ from few measurements $M$. To solve the undetermined system and approximate $x$ coefficients, the problem is considered as an optimization problem. Various recovery algorithms have been developed to solve the following optimization problem

$$
\tilde{\mathrm{x}}=\arg \min _{\mathrm{y}=\Phi \mathrm{x}}\|\mathrm{x}\|_{1} \quad \text { Subject to } \quad \mathrm{y}=\Phi * \mathrm{x}(4)
$$

where the recovered signal $\tilde{x}$ is the sparsest solution from many possible solutions of the optimization problem. $\|\mathrm{x}\|_{1}=\sum_{\mathrm{i}}\left|\mathrm{x}_{\mathrm{i}}\right|$ is the $\mathcal{L}_{1}$ norm of $x$ and represents the sum of the absolute values of $x$ coefficients. $\|x\|_{m}=\sqrt[m]{\sum_{i}\left|x_{i}\right|^{m}}$ is the $\mathcal{L}_{\mathrm{m}}$ norm $[34,38]$.

Considering noise, two cases are raised, noisy case and noiseless case. In practical applications, a random noise is added to the captured measurements during the signal processing. Hence, with the noisy measurements, equation (2) can be reformulated as

$$
\mathrm{y}=\Phi * \mathrm{x}+\mathrm{e}
$$

where $e$ is a random noise vector that needs to be estimated during the recovery process. Taking noise into account, the optimization problem can be reformulated as

$$
\tilde{\mathrm{x}}=\arg \min _{\mathrm{y}=\Phi \mathrm{x}+\mathrm{e}}\|\mathrm{x}\|_{1} \quad \text { Subject to } \quad \mathrm{y}=\Phi * \mathrm{x}+\mathrm{e}
$$


A number of reconstruction algorithms have been proposed to solve the linear programming problem, such as $\mathcal{L}_{1}$ norm minimization, gradient descent, iterative thresholding, matching pursuit, and orthogonal matching pursuit [15, 33,39-43]. Example of techniques that reconstructs the original signal with noise is described in [44].

For efficient compressive sensing, some requirements and assumptions should be taken into account before processing. Determining the sampling matrix to use for compression and which solver to apply depend on some conditions including sparsity, restrict isometry property, coherence, and measurements number.

Sparse signal is a signal with limited number of non-zero elements and most elements are zero or with very low power. A signal with $N$ samples is $k$-sparse means that it has only $k$ non-zero coefficients and $(N-k)$ zero elements, where $N$ is greater than $k$. Sparse representation is a major requirement for compressive sensing, it ensures the signal compressibility. It consists in presenting the signal in some domain with only essential information acquired.

The restricted isometry property is a property that characterizes orthonormal matrices. A matrix that satisfies this property in order $\mathrm{k}$ implies that

$$
\exists \delta \in(0,1) /(1-\delta)\|x\|_{2}^{2} \leq\|\Phi x\|_{2}^{2} \leq(1+\delta)\|x\|_{2}^{2}
$$

where $\delta$ is the restricted isometry constant (RIC)[32]. This property permits to guarantee the uniqueness of the recovered solution $\tilde{x}$, and it has to be considered during the matrix design. When a matrix satisfies the restricted isometry property, it guarantees that the undetermined system solution is unique and robust. Fourier, random Gaussian, and Bernoulli matrices are examples of matrices that satisfy the restricted isometry property [45].

For coherence property, it examines the sensing matrix quality and evaluates its efficiency. Mutual coherence of two matrices $\Phi$ and $\varphi$ measures the maximal correlation between any two elements of them, $\mu(\Phi, \varphi)$ is computed as

$$
\mu(\Phi, \varphi)=\sqrt{\mathrm{N}} \max _{1 \leq \mathrm{i}, \mathrm{j} \leq \mathrm{N}}\left|\left\langle\Phi_{\mathrm{i}} \mid \varphi_{\mathrm{j}}\right\rangle\right|
$$

where $1<\mu(\Phi, \varphi)<\sqrt{N}$. High coherence is equivalent to high correlation between $\Phi$ and $\varphi$ elements, which implies that the compressive sensing process needs more measurements. Compressive sensing requires that $\Phi$ is incoherent with $\varphi$. The small value of coherence means few measurements are needed for signal reconstruction. The coherence of the sensing matrix can be defined also as $\mu(\Phi)$, which represents the largest value of correlation between any two normalized columns of $\Phi, \mu(\Phi)$ is given as

$$
\mu(\Phi)=\max _{1 \leq \mathrm{i} \neq \mathrm{j} \leq \mathrm{N}}\left|\left\langle\Phi_{\mathrm{i}} \mid \Phi_{\mathrm{j}}\right\rangle\right|(9)
$$

where $\Phi_{\mathrm{i}}$ and $\Phi_{\mathrm{j}}$ represent two columns of $\Phi$. The smaller number of measurements necessitates the lower value of coherence [32].

\section{Classification of compressive sensing techniques}

Compressive sensing techniques can be classified into two main categories: distributed compressive sensing (DCS) and jointly compressive sensing (JCS) [35]. Distributed compressive sensing is done using two processes. It is based on sampling separately and recovering jointly. In other words, the measurements are acquired independently by each node. Each node samples its signal $x_{i}$ using its sensing matrix $\Phi_{i}$ to get its measurements signal $y_{i}$. The same reconstruction algorithm is then applied jointly to recover all the original signals $x_{i}$, where $i$ $=1 \ldots J, J$ is the number of nodes [46]. DCS enables distributed sampling of the received signals to reduce data 

those described in $[47,48]$.

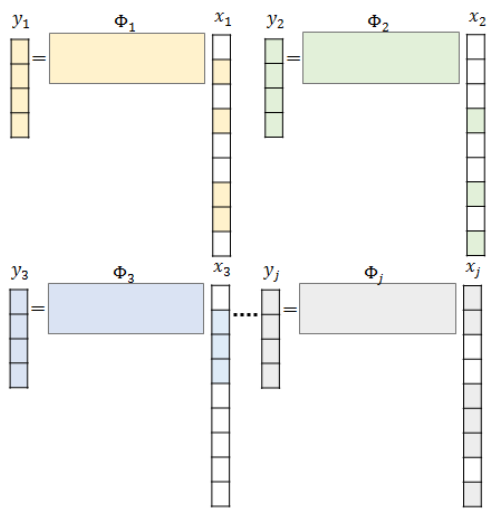

Fig. 3. Distributed compressive sensing.

Jointly compressive sensing (JCS) is based on the joint concept, in which measurements and reconstruction processes are done in the same process with the same sensing matrix. It permits to reconstruct jointly sparse signals. Examples of techniques in this category include those described in [47, 48]. Since the same compressive sensing techniques are involved for both categories, the classification of techniques will be based on the process involved. Some of sampling matrix techniques and recovery techniques are illustrated in the Fig. 4.

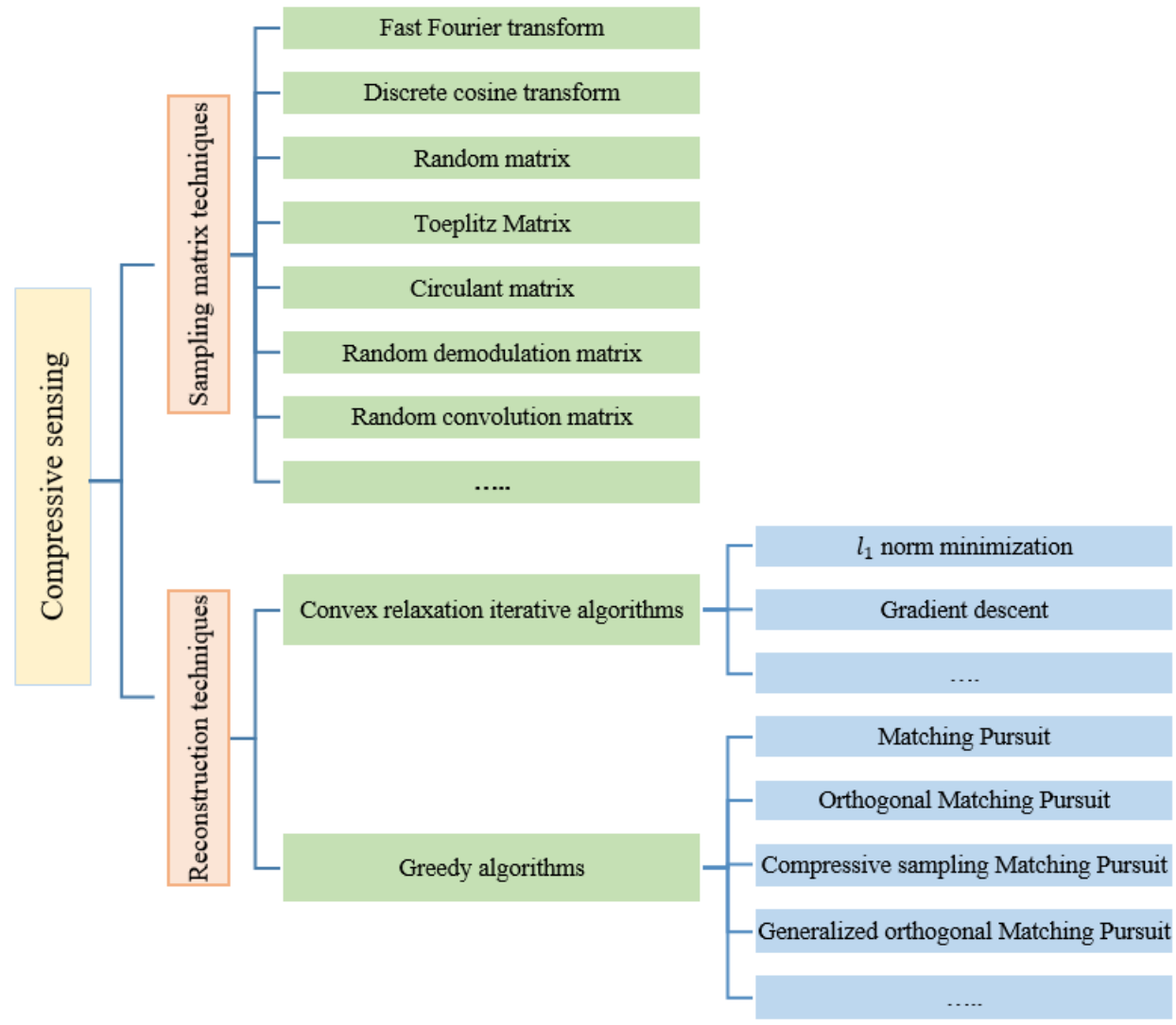

Fig. 4. Classification of compressive sensing techniques. 

below the Nyquist rate. It is designed to reduce the number of samples as much as possible while preserving the important information included in the original signal without losing any important data after recovery stage. It must be known, independent of the original signal, stable, incoherent with the sparsity basis matrix, and satisfies the RIP. Several matrices are used in compressive sensing as a sensing matrix, which respect the compressive sensing requirements.

194 For efficient compressive sensing, suitable sensing matrix is needed. Thus, the matrix choice is extremely important. Determining which matrix to use to acquire and reconstruct the signal at the receiver is a fundamental process. It might be based on some criteria including recovery complexity, speed, universality, number of measurements required, and hardware cost. Universality means that the signal can be sparse in any domain or any basis. Some of the most used sensing matrices include matrix randomly sampled from Fourier transform or Walsh-Hadamard transform, random matrix, Toeplitz matrix, Circulant matrix, random demodulation matrix, Gaussian or Bernoulli matrix, deterministic matrix, random convolution matrix, and discrete cosine transform. These sensing matrices are described and compared in Table I. Other sensing matrices have been proposed as extension of the listed matrices [31-33, 49-55].

Table 1 Compressive sensing matrices comparison

\begin{tabular}{|c|c|c|c|}
\hline Matrix & Concept & Strengths & Weaknesses \\
\hline $\begin{array}{l}\text { Fourier transform or Walsh- } \\
\text { Hadamard transform }[31,49]\end{array}$ & $\begin{array}{l}\text { Randomly selected rows of a discrete } \\
\text { Fourier transform matrix (DFT) }\end{array}$ & $\begin{array}{l}\text {-Easy to implement } \\
\text {-Fast recovery }\end{array}$ & $\begin{array}{l}\text {-Not universal } \\
\text {-High uncertainty } \\
\text {-Not accurate }\end{array}$ \\
\hline Random matrix [50] & Random Projection & $\begin{array}{l}\text {-Recovery with high efficiency } \\
\text {-Easy to implement } \\
\text {-Universal }\end{array}$ & $\begin{array}{l}\text { - } M \text { knowledge required } \\
\text {-More memory required }\end{array}$ \\
\hline $\begin{array}{l}\text { Toeplitz matrix, Circulant } \\
\text { matrix [51-53] }\end{array}$ & $\begin{array}{l}\text { The entries are independently distributed } \\
\text { in one row }\end{array}$ & $\begin{array}{l}\text {-Randomness is reduced } \\
\text {-Fast acquisition and recovery }\end{array}$ & Not universal \\
\hline $\begin{array}{l}\text { Random demodulation matrix } \\
{[21,50]}\end{array}$ & $\begin{array}{l}\text { Pseudorandom binary sequences are used } \\
\text { to modulate the input signal }\end{array}$ & $\begin{array}{l}\text {-Easy to implement } \\
\text {-Works in noiseless and noisy } \\
\text { signals } \\
\text {-Universal }\end{array}$ & $\begin{array}{l}\text {-High Uncertainty } \\
\text {-Not accurate }\end{array}$ \\
\hline $\begin{array}{l}\text { Gaussian or Bernoulli matrix } \\
{[32,54]}\end{array}$ & $\begin{array}{l}\text { Independent and identically distributed } \\
\text { (i.i.d) }\end{array}$ & $\begin{array}{l}\text {-Simple and easy to implement } \\
\text { - Universal }\end{array}$ & $\begin{array}{l}\text {-Costly hardware } \\
\text { implementation }\end{array}$ \\
\hline Deterministic matrix [55] & Deterministic matrix & $\begin{array}{l}\text {-Fast } \\
\text {-Reduced memoryusage }\end{array}$ & $\begin{array}{l}\text {-Requireshigh number of } \\
\text { measurements }\end{array}$ \\
\hline $\begin{array}{l}\text { Random convolution matrix } \\
{[21,50]}\end{array}$ & Convolution product & $\begin{array}{l}\text {-Simple and easy to implement } \\
\text {-Universal }\end{array}$ & $\begin{array}{l}\text {-Can only recover noiseless } \\
\text { signals } \\
\text {-Morenumber of measurements } \\
\text { required }\end{array}$ \\
\hline $\begin{array}{l}\text { Discrete cosine transform } \\
\text { (DCT) }[31,33]\end{array}$ & $\begin{array}{l}\text { Matrix formed from a random subset of } \\
M \text { rows }\end{array}$ & Simple and easy to implement & $\begin{array}{l}\text {-More } M \text { required } \\
\text {-Missing data recovery }\end{array}$ \\
\hline
\end{tabular}

205 As one can see from Table I, random matrices and matrices formed from random subset of DFT or DCS are 206 slowbut easy to implement. Toeplitz matrices and Circulant matrices are able to reduce the randomness 207 compared to others. Gaussian and Bernoulli matrices are also simple to implement, but theirhardware 

expected threshold, but random convolution matrices require less measurements.

\subsection{Recovery algorithms}

211 Recovery algorithms permit to reconstruct a compressed signal from few measurements $M$. The use of these 212 algorithms implies that the restrict isometry and coherent properties are satisfied. A number of recovery

213 algorithms have been proposed to reconstruct the original signal by solving the previously described optimization 214 problem. Recovery algorithms can be classified into two classes: convex relaxation iterative algorithms [15, 29, 215 31, 43, 44], and Greedy algorithms [29, 35, 40, 41, 43], as illustrated in Fig. 5. Other techniques have been 216 proposed as a combination of techniques from both categories [56-61].

\section{3.2.1 Convex relaxation iterative algorithms}

218 The convex relaxation category is the process used to solve the undetermined system via linear programming 219 system. Some of the convex techniques include $\mathcal{L}_{0}$ norm minimization, $\mathcal{L}_{1}$ norm minimization, and $\mathcal{L}_{2}$ norm minimization. Since $\mathcal{L}_{0}$ norm represents the number of non-zero elements of the signal, this solution can recover the sparse signal, but according to Donoho $[15,61]$, this solution is a non-deterministic polynomial-time hard (NP-hard) problem. The $\mathcal{L}_{2}$ norm is not also considered, because it represents the energy of the signal and it cannot find the sparsest solution. Donoho then suggested $\mathcal{L}_{1}$ norm minimization as the better solution. It represents the sum of absolute values of all signal elements and needs to solve the convex problem

$\tilde{\mathrm{x}}=\arg \min _{\mathrm{y}=\Phi \mathrm{x}}\|\mathrm{x}\|_{1} \quad$ Subject to $\quad\|\mathrm{y}-\Phi \mathrm{x}\|_{2} \leq \epsilon$ where $\epsilon$ denotes the amount of noise in the measurements. Taking $\epsilon$ into account, basis pursuit (BP) and basic pursuit de-noisy are two examples of $\mathcal{L} 1$ norm minimization algorithm, where $\epsilon=0$ and $\epsilon \neq 0$ respectively. The $\mathcal{L} 1$ norm minimization algorithm requires that the sensing matrix satisfies the restrict isotropy property with a small value of RIC. It can recover the original signal via linear programing and can find the compressible solution with high probability. Considering the basic pursuit solution, equation (10) can be reformulated as $\tilde{\mathrm{x}}=\min \|\mathrm{y}-\Phi \mathrm{x}\|_{2}+\gamma\|\mathrm{x}\|_{1}(11)$ Basic pursuit minimizes the cost function, $\gamma$ is a positive constant. The algorithm operates in an iterative process, step by step. Firstly, the first guest is initialized $\left(x=x_{0}=\Phi^{\prime} y\right)$, which represents the minimal energy of the signal, then, the cost function ( $\left.\min \left\|y-\Phi x_{0}\right\|_{2}\right)$ is provided. Secondly, for $\mathrm{k}$ iterations, the new sensing matrix is computed by selecting the required measurements number $M$, and the signal coefficients are modified to attend the minimized form. The algorithm works iteratively until obtaining enough signal coefficients less than its sparsity level. Other convex iterative relaxation algorithms have been proposed such as gradient descent, and iterative thresholding $[39,40,62]$. This class of recovery algorithms can work with any matrix that satisfies the restrict isometry property.

\subsubsection{Greedy algorithms}

241 Greedy algorithms consist on selecting one position of a non-zero element of $x$. It is equivalent to pick one 242 column from the sensing matrix. A number of techniques have been proposed under this category. Examples of 243 these techniques are matching pursuit (MP) [63], orthogonal matching pursuit (OMP) [62], stage wise orthogonal matching pursuit (StOMP) [56], compressive sampling matching pursuit (CoSaMP) [57], generalized orthogonal matching pursuit (GOMP) [58, 59], and regularized orthogonal matching pursuit (ROMP) [41, 43]. Matching 
246 pursuit algorithm is the basic solver and the others are inspired from it to handle some of its limitations. Other

247 Greedy algorithms include iterative hard thresholding (IHT) [64], normalized iterative hard thresholding (NIHT)

248 [65], hard thresholding pursuit (HTP) [66], and normalized hard Thresholding pursuit (NHTP) [67].

249 Matching pursuit algorithm permits to recover the signal by decomposing it into a linear expansion of waveforms

250 selected from a dictionary. It consists in selecting a column from A that maximizes the inner product of the

251 current residual. Firstly, the vector that corresponds to the longest projection of $x$ is selected from the dictionary.

252 Secondly, the signal $x$ is orthogonalized by removing any element of the selected vector from $x$ to get the residual

253 of $x$, that has the lowest energy. Then, the two previous steps are repeated to the remaining of the dictionary in an

254 iterative process until the residual norm is low than a threshold $c$. The residual norm of $x$ after $\mathrm{t}$ steps, denotes $r_{t}$,

255 satisfied

256

$$
\left\|\mathrm{r}_{\mathrm{t}}\right\| \leq \mathrm{c} / \sqrt{\mathrm{t}}
$$

257 Orthogonal matching pursuit algorithm is inspired from matching pursuit algorithm by removing not only 258 elements of the selected vector from $x$, but also from the basis before repeating the process. It is proved that orthogonal matching pursuit presents best results solution than matching pursuit, but it is more expensive. GOMP algorithm is the generalized version of orthogonal matching pursuit technique. It consists of selecting multiple indices in each iteration instead of one.

262 Greedy techniques are known as the faster techniques to recover a sparse signal. However, the recovered signal 263 has been shown to be not optimal, and cannot be considered as the exact recovered signal [68]. Because in 264 practical situations the original signal is not known, it is difficult to evaluate the efficiency of this type of 265 algorithms by comparing the original signal to the output of the algorithm.

\subsubsection{Recovery algorithms comparison}

267 Each category has its features and requirements. Convex algorithms are consistent and Greedy algorithms are 268 fast. They are complementary to each other, but they share some characteristics. Table II presents a comparison between six reconstruction techniques in terms of complexity and minimum measurements number required forreconstruction $[69,70]$.For most of these techniques, the reconstruction complexity depends on the number of samples $N$, sparsity level $k$, and measurements number $M$.The reconstruction complexity of convex relaxation algorithms, such as $\mathcal{L}_{1}$ norm minimization, is very high compared to the Greedy algorithms, which makes them not practical for hardware implementation. However, algorithms under this category require less measurementscompared to the Greedy algorithms, which makes them faster to process.

Table II Solvers comparison based on complexity and measurements number

\begin{tabular}{|l|c|c|}
\hline \multicolumn{1}{|c|}{ Reconstruction techniques } & Measurements number & Complexity \\
\hline $\mathcal{L}_{1}$ norm minimization & $\mathrm{O}(\mathrm{k} \log \mathrm{N})$ & $O\left(N^{3}\right)$ \\
\hline Orthogonal matching pursuit & $\mathrm{O}(\mathrm{k} \log \mathrm{N})$ & $\mathrm{O}(\mathrm{kMN})$ \\
\hline Stage wise orthogonal matching pursuit & $\mathrm{O}(\mathrm{N} \log \mathrm{N})$ & $\mathrm{O}(\mathrm{N} \log \mathrm{N})$ \\
\hline Regularized orthogonal matching pursuit & $\left(k \log ^{2} N\right)$ & $\mathrm{O}(\mathrm{kMN})$ \\
\hline Compressive sampling matching pursuit & $\mathrm{O}(\mathrm{klog} \mathrm{N})$ & $\mathrm{O}(\mathrm{MN})$ \\
\hline
\end{tabular}


Table III summarizes the comparisons between the two solvers categories in terms of speed, measurements, and complexity. Unlike Greedy algorithms, convex relaxation algorithms require small number of measurements for efficient reconstruction; however, techniques under this category are time consumingand complex in terms of computation. Greedy techniques are not efficient, but they are easy to implement.

Table IIISolvers categories comparison

\begin{tabular}{|c|c|}
\hline \begin{tabular}{|c|} 
Convex relaxation algorithms \\
Can be trusted for recovering sparse solutions given sufficient measurements
\end{tabular} \\
\hline \multicolumn{2}{|c|}{ Guarantee stable recovery } \\
\hline Require few measurements & Require more measurements for perfect reconstruction \\
\hline Complex & Sample and easy to implement \\
\hline More accurate & Not accurate \\
\hline High procesing time & Low proceing time \\
\hline
\end{tabular}

281

282

283

284

285

286

287

288

289

290

291

292

293

294

295

296

297

298

299

300

301

302

303

304

305

Other solvers have been proposed to combine the strengths of both categories. In [46, 47, 60, 71], Bayesian compressive sensing is proposed as a reconstruction technique to estimate unknown parameters [72]. It is shown that this technique is fast and provides minimal errors compared to other techniques [46, 47, 60, 72]. Sparse signal reconstruction from multiple measurements vectors (MMVs) is also another proposed recovery technique [60], which is based on sparse Bayesian learning to solve the compressive sensing problem. It is shown that MMVs gives good results compared to recovery from single measurement vectors (SMVs) approach. In [73], fusion based compressive sensing is proposed as a reconstruction technique that combines multiple recovery techniques.

\section{Compressive sensing:Applications and challenges}

\subsection{Compressive sensing schemes}

Compressive sensing schemes illustrate how the compressive sensing operates, and how the signal is sampled and acquired simultaneously. Classical ADC works on the Shannon-Nyquist theorem, which limits their utilization and efficiency. Thanks to compressive sensing, which is a revolution of the sampling theorem and digital signal processing filed. The compressive measurements concept is used for signal processing as a new concept called compressive signal processing CSP [74]. Signal acquisition can be done in a very short time acquiring only the essential information of the sparse signal. Many schemes have been proposed for compressive sensing implementation to replace the classical ADC. Examples of these schemes include random demodulator [31], modulated wideband converter [31], random filtering [75, 76], random convolution [77, 78], and compressive multiplexer [79].

Random demodulator is an acquisition scheme, known also as the analog to information converter [31]. Its architecture is illustrated in Fig. 5. This technique assumes that the original signal is sparse in some domain. It is a multiplication block followed by a pass-low filter, in which the signal is modulated by multiplying it by a high speed sequence generated by a pseudo random generator in the analog domain. The low-pass filter output is then sampled at rate lower than Nyquist rate. Finally, a recovery algorithm is applied the receiver to reconstruct the input signal $[80,81]$. This scheme aims to process signals in time domain. 


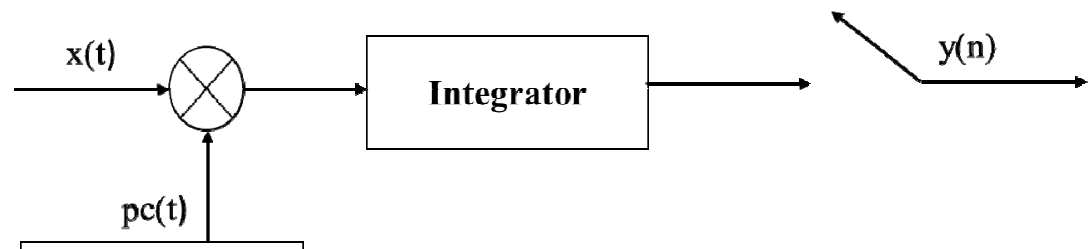

\section{Pseudorandom generator}

Fig. 5. Random demodulator scheme.

A modulator wideband converter is a compressive sensing scheme used for multiband sparse signals. In contrast to the random demodulator scheme, the modulator wideband converter can also work in frequency domain [31]. Its scheme is illustrated in Fig. 6.

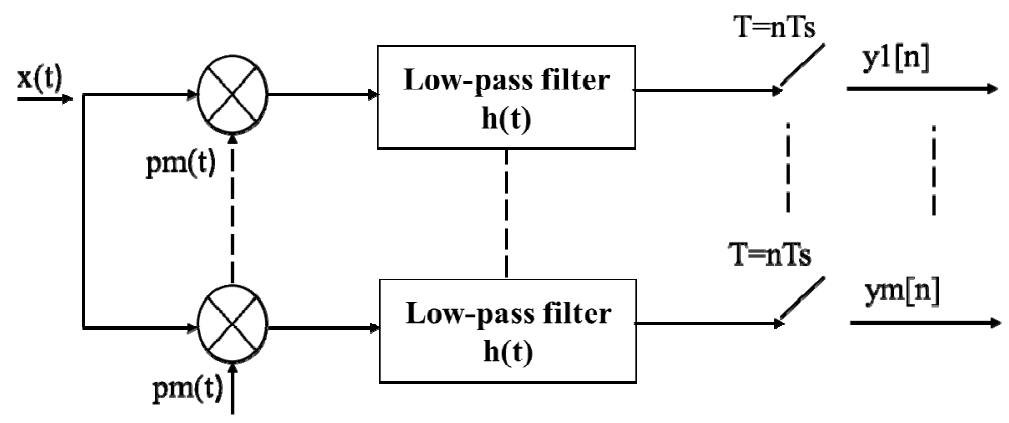

Fig. 6. Modulator wideband converter scheme.

For $m$ channels, the original signal is multiplied by a periodic waveform $p_{i}(t)$, a low pass filter $h(t)$ is then applied, the output sequences $y_{i}[\mathrm{n}]$ are used as input for the recovery algorithm block. Modulated wideband converter is known also as Xampling [81].

Random filtering is a generic and structure architecture based on the finite impulse response (FIR) filter. In time domain, the sparse signal is acquired through the convolution with a random tap FIR filterh. This scheme can be used to capture and recover sparse signals when the number of the random taps is known. The random tap can be obtained by using Gaussian or Bernoulli distribution with mean zero and variance 1. This scheme has the potential to be implemented for new ADCs. It is easy to implement in hardware or software. It can also be used for continuous time signal and streaming [75, 76].Fig. 7 shows the processes of the random filtering scheme.

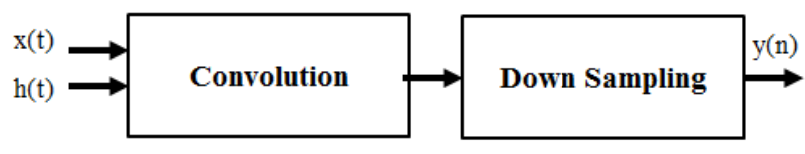

Fig. 7. Random filtering scheme in time domain.

Random filtering in frequency domain is called random convolution and it is based on fast Fourier transform (FFT). The sparse signal is captured through the convolution with a random pulse or random filterh. This process is then followed by a random subsampling. Despite the randomness, this scheme is universal and it represents fast computations by using double FFT [77, 78]. Fig.8 illustrates the processes of the random convolution scheme. 


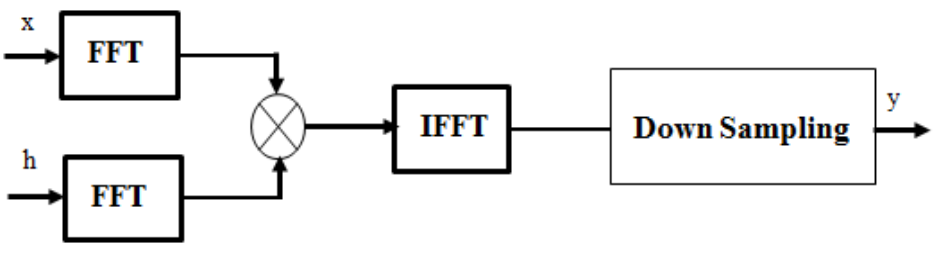

Fig. 8. Random convolution scheme in frequency domain.

Another compressive sensing scheme, called compressive multiplexer, was proposed by the authors of [79]. This techniqueis mostly used for multi-channel compressive sensingand it requires one ADC for all the channels instead of one ADC by channel, as shown in Fig. 9.It consists of coding each channel with an orthogonal code $p_{i}$ where $i=1 \ldots l, l$ is the number of channels. All the coded channels are summed and sampled through the ADC. The code is a pseudo random chipping sequence $(\mp 1)$.

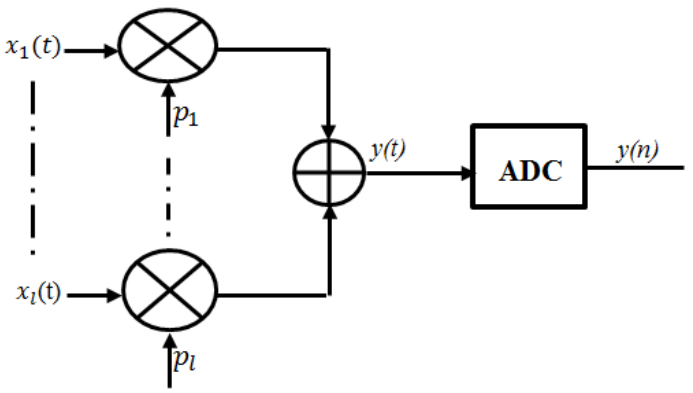

Fig. 9.Compressive multiplexer scheme.

The compressive sensing architectures discussed above have been tested and implemented in testbed environments, where it was demonstrated that these schemes can sample the sparse signals in practical environments [82]. The implementation of compressive sensing in hardwarestill represents a great challenge. Thus, schemes need to be developed and should be applicable to real scenarios not only limited to simulations.

\subsection{Compressive sensing applications}

\section{2. 1 Cognitive radio networks}

Over the years, compressive sensing has attracted the attention of many researchers who applied itfor wide band spectrum sensing and channel estimation in the context of cognitive radio and MIMO[12, 42, 71, 83-101]. In cognitive radio, because of the spectrum underutilization, sparsity condition is valid and transmitted signals are compressible. Several compressive sensing approaches that focus on recovering the signal have been proposed [42, 83-88]. In [83], the authors proposed to detect the presence or absence of the primary user (PU) signal from a minimal number of measurementsinstead of using the entire SU received signal. Thus, the cognitive radio model for spectrum sensing can be reformulated in terms of compressive sensing as follows

H0: $y=\Omega n, \quad$ PU absent

$\mathrm{H} 1: \quad y=\Omega(S+n), \quad$ PU present 
where $y$ is the compressive measurements that contain only the significant information for detection, $n$ is the additive white Gaussian noise, $\Omega$ is a known sensing matrix $\left(M^{*} N\right)$ with $(M<<N), S$ is the signal to be detected, $\mathrm{H} 0$ denotes the PU signal is absent, and $\mathrm{H} 1$ denotes the PU signal is present. The test statistic $T$ is deduced by applying the likelihood ratio test and given as follows

$T=y^{T}\left(\Omega \Omega^{T}\right)^{-1} \Omega S(13)$

The test statistic corresponds to the compressive detector and contains sufficient information to decide about the spectrum availability. Tis then compared with an appropriate threshold for decision making. In [84], compressive sensing approach is extended to spectrum sensing and PU localization problem in a collaborative compressive sensing approach. The proposed model is implemented with Kalman filter for PU tracking and dynamic spectrum sensing for estimating the spectrum utilization. In [85], a wideband analog signal is acquired directly in the analogic domain using an analog to information converter. In [86, 87], an adaptive sequential compressive sensing approach is proposed to detect white spaces in the radio spectrum with a minimum number of measurements. This approach aims to deal with the noise uncertainty in order to allow SU to detect PU presence even at low SNR. In [88], a wideband spectrum sensing is proposed, in which only a portion of the wideband spectrum is estimated by compressive sensing approach to reduce the memory size needed for storage and also reduce computational complexity.

Yet other techniques have been proposed that are based on Bayesian compressive sensing $[60,89]$. Bayesian compressive sensing is applied as a recovery algorithm to estimate the unknown parameters including the original signal and noise variance. In [90], a collaborative compressive spectrum sensing is proposed, which operates with multiple nodes for wideband sensing. Sensing is performing by multiple nodes in a distributed environment, each node performs based on its measurements and measurements from other nodes of the same band. In [91], a parallel spectrum sensing scheme is proposed, in which the received signal is compressed by a sensing matrix in a parallel structure. A wavelet edge detector is then applied to combine the outputs from each node. In [92], kronecker sparse basis is used to perform compressive spectrum sensing. In [93], compressive sensing framework is used as an acquisition system for spectrum sensing in cognitive radio without reconstructing the signal at the receiver, which is known as compressive signal processing. The authors argue that since the main objective of spectrum sensing is to sense the spectrum and decide about its state, it is not necessary to recover it after sensing and analyzing it. According to these authors, this objective is achieved based only on compressed measurements. The spectrum sensing problem is then reformulated based on known sensing matrix and required signal knowledge to determine the channel availability.

In [94], a robust cyclic compressive sensing technique is proposed for spectrum sensing in order to exploit the cyclic sparsity to reconstruct the sparse 2 -cyclic spectrum using $\mathcal{L}_{1}$ norm minimization. This technique aims to overcome the problem of high dimensional signals sampling by reconstructing only the cyclic spectrum instead of reconstructing the original signal and its frequency responses. In [95], the authors proposed an adaptive compressive sensing technique that adaptively adjusts the number of measurementsindependently of the sparsity level. In [96], compressive sensing has been applied for wideband power spectrum sensing, which allows sampling and reconstructing the power spectral density of each channelinstead of the signal. This proposed approach uses the multicoset sampling technique based on the minimal sparse ruler problem.In [97], a cooperative spectrum sensing technique is proposed based on the rank minimization of the measurements signal 
at the SU receiver. In [12], wavelet transform is used in order to directly scan the wide band spectrum using a high rate ADC to detect the edges of occupied bands. In [98], compressive sensing framework is implemented in each SU receiver to handle the problem of malicious users. This approach detectsand discards signals from malicious users based on low rank matrix completion.In [99, 100], a real time algorithm is proposed that combines compressive sensing with geo-location database at the SU receiver for spectrum sensing to decide about the band occupancy. In [101, 102], compressive sensing is applied in spectrum sensing to reduce the noise at the SU receiverand reduce the sensing overhead by sensing multiple narrow bands using selective filers.

In order to evaluate the performance of compressive sensing techniques, several metrics have been used, including:recovery success rate [103], failure rate [33], error sparsity, signal sparsity [104], reconstruction error, recovery time [71], mean square error [89], compression ratio, processing time [104], probability of returning the true solution and storage cost of the sensing matrix. Recovery success rate represents the success rate of the recovery algorithm and who much more successfulthe algorithm is among a number of experiments. It aims to calculate the similarity between the reconstructed signal and the original signal for several values of $k, M$ and $N$. Failure rate represents the opposite role of recovery success rate. It calculates the number of time the signal is not reconstructed exactly among a number of experiments. Error sparsity and signal sparsity are two metrics considered to investigate the sparsity level of the estimated signal after reconstruction, compare it with the sparsity level of the original signal before compressive sensing, and calculate the error sparsity. Reconstruction error represents the error level of the reconstruction algorithm. It is calculated as the normalized value of the difference between the reconstructed signal and the original signal. Recovery time evaluates how faster the recovery algorithm is. Processing time evaluates how faster the technique is taking into account the three stages of compressive sensing technique. Compressive ratio $(M / N)$ represents the ratio between the sample number of $x$ and the sample number of the measurements. It aims to make sure that a signal with huge number of sample can be reconstructed from few measurements.

\section{2. 2 MIMO}

In addition, compressive sensingis applied toMIMO based cognitive radio systems to enhance these systems [105-107]. In [105], the authors proposed a new architecture of the receiver for MIMO-OFDM in order to sample multi-channel signals using a single ADC instead of using multiple ones.For different MIMO schemes $(2 \times 2,3 \times 3$, and $4 \times 4$ ), this approach allows mixing the transmitted symbols from each antenna, separating the transmitted symbols from each antenna and then recoveringthem from the mixed signals. It aims to reduce the sampling rate, sampling time, hardware cost, and the number of ADCs by exploring the diversity, the channel occupancy,and the sparsity of MIMO-OFDM channels. In [106], compressive sensing is used to perform the channel estimation with sparse multi-path MIMO signals. In [107], the authors proposed a novel architecture of MIMO-OFDM system that exploits compressive sensing by using a 4x4 MIMO scheme.Under the conventional MIMO-OFDM system, the number of the required ADCs corresponds to the number of antennas at the receiver. With the proposed system, the number of ADCsis reduced to one ADCand the MIMO detector is substituted by the compressive sampling reconstruction as shown in Fig. 10.

(a)

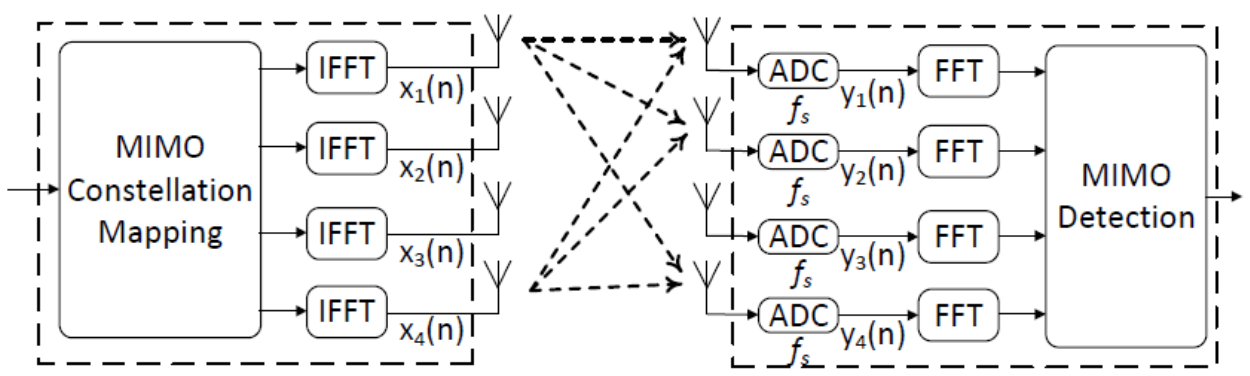


(b)
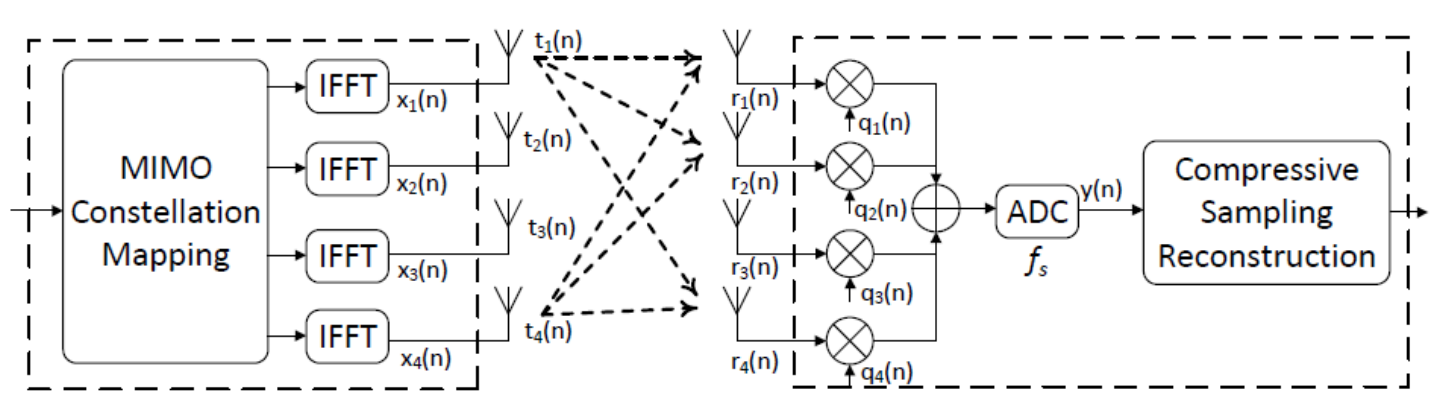

Fig. 10. (a) Conventional MIMO-OFDM system; (b) Proposed MIMO-PFDM based compressive sensing [105].

The sparse signals from multiple antennas are jointly combined and sampled. The reconstruction process reconstructs the entire signals directly on frequency domain and separates them [105].

\section{2. 3Channel estimation}

Another application of compressive sensing is channel estimation to identify which antenna is used for transmission and estimate the channel coefficients through the received signal. Channel estimation has been investigated in wireless communications to allow the receiver to estimate the impact of the channel on the signal [24].In [108], compressive channel estimation technique is adopted in MIMO communication systems for wideband in order to address the limitations of the conventional techniques. It allows estimating all channels responses $h_{i, j}$ between the transmitter $i$ and the receiver $j$.In [109], compressive sensing is used for pilot allocation in MIMO-OFDM systems to estimate the channel impulse response with reduced number of pilotsbased on the sparsity of channel impulse response.In [110], the authors proposed a new approach for channel estimation through compressive sensing, in which the sparsity feature of the signal is explored to estimate selective channels in OFDM systems. This approach aims also to estimate and overcome the interferences problems in multi-carrier communications whether inter-symbol or inter carrier interferences.In [111], the authors investigate channel estimation using compressive sensing and evaluate it based on Bit Error Rate and Mean Square Error.To estimate the channel response coefficients, OMP and SAMP algorithms have been applied for non-contiguous OFDM using reduced number of measurements.

In [112,], compressive sensing has been applied on a pilot aided channel estimation technique in order to overcome its limitations with the assumption that multipath channels are sparse. The authors showed that with compressive sensing, sparse multipath channels can be estimated with high accuracy and low pilot overhead using a fixed number of pilots for the estimation process. The authors suggested adopting banded channel matrix for the sensing process with unknown symbols data at the receiver. The model is tested for different channel types, including time variantand invariant channels.

\subsection{Compressive sensing limitations and challenges}

A number of issues and limitations have been investigated in order to make compressive sensing a suitable and efficient solution for the next generation of wireless communication systems. These issues and limitations are especially related to the implementation aspects for real scenarios. Examples of these limitations include 


\section{Conclusion}

497 In this paper, we have provided an in depth review of existing compressive sensing techniques. These designing practical sensing matrix, exact and approximate sparsity, hardware implementation, uncertainty due to the noisy and real environment, recovery uncertainty, and RIP proof $[82,113]$. The matrix design is one of main the limitations of compressive sensing techniques. Efficient compressive sensing requires designing a practical structured sampling matrix that can sample the signal and take only the essential information taking into account the cost and the speed for the encoding process. Implementing a random matrix in practical applications is challenging because of its unstructured nature and randomness in its elements [113]. Thus, the design is complex, costly, and requires high memory storage. Therefore, these limitations need to be addressed, and, thus represent open research topics to be investigated.

Regarding sparsity, compressive sensing requires signals to be sparse; however, signals can only be approximately sparse in practical scenarios [37, 82, 113, 114]. In [115], the authors showed that in practice the sparsity of a signal is unknown and needs to be estimated. They proposed a technique to measure the sparsity of the signal in terms of the $\mathcal{L}_{0}$ norm $\|x\|_{0}$ that represents the number of non-zero coefficients of the signal $x$. Thus, there is a need for techniques to transform signals into sparse signals in real applications.

Regarding the limitations due to noise, most of the proposed compressive sensing techniques consider the Gaussian noise with known or unknown variance. In addition to the noise, other factors impact compressive sensing techniques' performances such as interference, high level of noise uncertainty, channel uncertainty, and imperfections. Because these factors, compressive sensing performance degrades in real scenarios $[66,82,113]$. However, a few papers investigated the impact uncertainty due to the noise in measurements. Thus, there is a great need for investigating and developing practical compressive sensing techniques that can deal with the imperfections of real networks. In addition, dealing with real signals involves non-linear signals, which is another issue that limits the application of compressive sensing for non-linear measurements [82]. Furthermore, hardware implementation of compressive sensing is also one of themain limiting challenges due to a number of problems, including synchronization, calibration, and the uncertainty in measurements $[82,113]$.

Research shows that it is not easy to select which compressive sensing technique to apply for a specific application since efficient and complex techniques require great deal of processing time [82, 113]. Indeed, compared to the other alternative acquisition and recovery techniques, compressive sensing approach is characterized by its efficiency in sampling high dimensional signals below Nyquist rate, which makesthe spectrum scanning faster that spectrum sensing techniques. It can also recover signals from few measurements with high efficiency and with low recovery errors. Designing fast ADCs is yet another solution for signal sampling in terms of hardware. However, ADCs are costly and they capture all the signal, which makes them slow and not efficient compared tothe compressive sensing solution. techniques were classified in categories according to the involved process: sampling matrix and recovery. We have also provided an overview of metrics for evaluating the efficiency of these techniques. A comparison of several compressive techniques was given and discussed. In addition, major applications of compressive sensing have been discussed such as spectrum sensing, channel estimation, and MIMO based cognitive radio. Compressive sensing is a promoting tool for the next generation of communication systems. However, it still faces a number of challenges. For example, in cognitive radio applications, compressive sensing faces 
considerable challenges to be conducted by researchers such as hardware design, application in real time systems, and cooperative and realistic scenarios. For sparsity representation, the challenge resides in how to demonstrate the sparsity of a given signal and how to transfer it to sparse when it is necessary. For sampling matrix, the challenge is to determine the suitable sampling matrix and how to address the complexity in hardware implementation. For reconstruction, the challenge resides in how to separate the target signal from additional signals in multi-user multi-channel environments, where the channel powers and behaviors evolve over time.

\section{ACKNOWLEDGMENT}

513

The authors acknowledge the support of NSF, grant \# 1443861, and the Fulbright program.

\section{References}

[1] T. Yucek and H. Arslam, A Survey of spectrum sensing algorithms for cognitive radio applications, IEEE Commun. Surv. Tutorials, $11(1)(2009) 116-130$.

[2] Y. Zeng, Y.C. Liang, A. T. Hoang, and R. Zhang, A review on spectrum sensing for cognitive radio: Challenges and solutions, EURASIP J. Adv. Signal Process. (2010) 1-16.

[3] H. Sun, A. Nallanathan, C. X. Wang, and Y. Chen, Wideband spectrum sensing for cognitive radio networks: a survey, IEEE Wirel. Commun. 20(2) (2013) 74-81.

[4] H. Sun, N. Arumugam, C. Shuguang and W. C. Xiang, cooperative wideband spectrum sensing over fading channels, IEEE Trans. Vehicular Tech., (2015).

[5] N. Gellerman, H. Reyes, and N. Kaabouch, Cognitive radio technology for improving the reliability of UAS/UAV networks, IEEE Proc. Aerospace Conf., (2015).

[6] R. A. Jani and Y. Sharma, A review on spectrum sensing for cognitive radio networks, Int. J. Adv. Eng. Res. Dev. 1(5) (2014) 5-8.

[7]K. Kim, Y. Xin, S. Rangarajan, Energy detection based spectrum sensing for cognitive radio: An experimental study, IEEE Global Telecommunications Conf., (2010) 1-6.

[8] F. Salahdine, H. E. Ghazi, N. Kaabouch, and W. F. Fihri, Matched filter detection with dynamic threshold for cognitive radio networks, Int. Conf. Wirel. Netw. Mob. Commun. (2015).

[9] M. Naraghi-Pour and T. Ikuma, Autocorrelation-based spectrum sensing for cognitive radios, IEEE Trans. Vehicular Technology, 59(2) (2010), 718-733.

[10] H. Reyes, S. Subramaniam, and N. Kaabouch, A Spectrum sensing technique based on autocorrelation and Euclidean distance and its comparison with energy detection for cognitive radio networks, Elsevier Computers \& Electrical Engineering J., (2015).

[11] S. Subramaniam, H. Reyes, and N. Kaabouch, Spectrum occupancy measurement: an autocorrelation based scanning technique using USRP, IEEE 16th Annual Wirel. Microwave Techno. Conf. (2015) 1-5.

[12] Z. Tian, Y. Tafesse, and B. M. Sadler, Cyclic feature detection with sub-Nyquist sampling for wideband spectrum sensing, IEEE J. Sel. Signal Process., 6(1) (2012) 58-69.

[13] Z. Tian and G. B. Giannakis, A wavelet approach to wideband spectrum sensing for cognitive radios, Proc. International Conf. on Cognitive Radio Oriented Wirel. Networks and Comm., (2006) 1-5.

[14] I. F. Akyildiz, B. F. Lo, and R. Balakrishnan, Cooperative spectrum sensing in cognitive radio networks: A survey, Phys. Commun. ELSEVIER, 4(1) (2011) 40-62.

[15] D. L. Donoho, Compressed sensing, IEEE Trans. Inf. Theory, 52(4) (2006) 1289-1306.

[16] E. J. Candès, J. Romberg, and T. Tao, Robust uncertainty principles: Exact signal reconstruction from highly incomplete frequency information, IEEE Trans. Inf. Theory 52(2) (2006) 489-509.

[17] E. J. Candès, Compressive sampling, Proc. Int. Congr. Math., vol. 3, pp. 1433-1452, 2006.

[18] J. H. G. Ender, On compressive sensing applied to radar, An Int. J. Signal Process. ELSEVIER, 90(5) (2010) 1402-1414.

[19] M. Lustig, D. Donoho, and J. M. Pauly, Sparse MRI: The application of compressed sensing for rapid MR imaging, Magn. Reson. Med., vol. 58, no. 6, pp. 1182-1195, 2007.

[20] M. Lustig, D. L. Donoho, J. M. Santos, and J. M. Pauly, Compressed sensing MRI, IEEE Signal Process. Mag. (2007) $72-82$.

[21] W. Chen and I. J. Wassell, Energy efficient signal acquisition in wireless sensor networks : A compressive sensing Framework, 6th Int. Symp. Wirel. Pervasive Comput. (2011) 1-6.

[22] M. Morelli, and U. Mengali, A comparison of pilot-aided channel estimation methods for OFDM systems, IEEE Trans. Signal Processing, 49(12) (2001) 3065-3073.

[23] O. Edfors, M. Sandell, J. J. van de Beek, S. K. Wilson, and P. O. Borjesson, OFDM channel estimation by singular value decomposition, IEEE Trans. Commun., 46 (1998) 931-939.

[24] R. Negi and J. Cioffi, Pilot tone selection for channel estimation in a mobile OFDM system, IEEE Trans. Consum. Electron, 44 (1998) $1122-1128$.

[25] M.C. Necker and G. L. Stüber, Totally blind channel estimation for OFDM on fast varying mobile radio channels, IEEE TranS. Wireless Communications, 3(5) (2004) 1514-1525.

[26] S. Colieri, M. Ergen, A. Puri and Bahai A, A study of channel estimation in OFDM systems, IEEE Conf. Proceed. Vehicular Technology, 2 (2002) 894-898.

[27] W. U. Bajwa, J. Haupt, A. M. Sayeed and R. Nowak, Compressed channel sensing: a new approach to estimating sparse multipath channels, IEEE Proceedings, 98(6) (2010) 1058-1076.

[28] E. Candes and J. Romberg, Sparsity and incoherence in compressive sampling, Inverse Probl., 23(3) (2006) 969-985. 
[29] E. J. Candes and M. B. Wakin, An introduction to compressive sampling, IEEE Signal Process. Mag. 25(2) (2008) 21-30

[30] R. G. Baraniuk, E. Candes, M. Elad, and Y. Ma, Applications of sparse representation and compressive sensing [scanning the issue], IEEE Proceeding 98(6) (2010) 906-909.

[31] Z. Han, H. Li, and W. Yin, Compressive sensing for wireless networks, CAMBRIDGE (2013).

[32] B. Bubacarr and J. Tanner, Improved bounds on restricted isometry constants for Gaussian matrices, J. Matrix Anal. Appl., 31(5) (2010) 2882-2898.

[33] V. Tiwari, P. P. Bansod, and A. Kumar, Designing sparse sensing matrix for compressive sensing to reconstruct high resolution medical images, Cogent Eng. (2015).

[34] E. J. Candes and T. Tao, Decoding by linear programming, IEEE Trans. Inf. Theory 51(12) (2005) $4203-4215$.

[35] Meenakshi and S. Budhiraja, A Survey of compressive sensing based Greedy pursuit reconstruction algorithms, Int. J. Image, Graph. Signal Process. (2015) 1-10.

[36] D. Baron, M. F. Duarte, M. B. Wakin, S. Sarvotham, and R. G. Baraniuk, Distributed compressed sensing, Eprint arXiv (2009).

[37] Z. Zhang, S. Member, Y. Xu, and S. Member, A survey of sparse representation: Algorithms and applications, IEEE Access, 3(2015) 490-530.

[38] E. Candes, J. Romberg, and T. Tao, Stable signal recovery from incomplete and inaccurate measurements, Commun. Pure Appl. Mathematics (59) (2006)1207-1223.

[39] R. Jin, T. Yang, and S. Zhu, A New analysis of compressive sensing by stochastic proximal gradient descent, arXiv, 2 (2013) 1-8.

[40] M. Masood and T. Y. Al-Naffouri, Sparse reconstruction using distribution agnostic bayesian matching pursuit, IEEE Trans. Signal Process., 61(21) (2013) 5298-5309.

[41] W. Dai and O. Milenkovic, Subspace pursuit for compressive sensing signal reconstruction, IEEE Trans. Inf. Theory 55(5) (2009) 2230-2249.

[42] J. Chen, L. C. Jiao, J. Wu, and X. Wang, Compressive spectrum sensing in the cognitive radio networks by exploiting the sparsity of active radios, Wirel. Networks, 19(5) (2013) 661-671.

[43] D. Needell and J. a. Tropp, CoSaMP: Iterative signal recovery from incomplete and inaccurate samples, Appl. Comput. Harmon. Anal. 26(3) (2009) 301-32.

[44] T. T. Cai and L. Wang, Recovery with noise, IEEE Trans. Inf. Theory 57(7) (2011) 4680-4688.

[45] M. M. Abo-zahhad, A. I. Hussein, and A. M. Mohamed, Compressive sensing algorithms for signal processing applications : A Survey, Sci. Res. Publ. (2015)197-216.

[46] D. Baron, M. F. Duarte, M. B. Wakin, S. Sarvotham, and R. G. Baraniuk, Distributed compressed sensing, Eprint arXiv, (2009).

[47] S. D. Babacan, R. Molina, and A. K. Katsaggelos, Bayesian compressive sensing using Laplace priors, 19(1) (2010) $53-63$.

[48] D. Baron, S. Sarvotham, and R. G. Baraniuk, Bayesian compressive sensing via belief propagation, arXiv, (2009) 1-25.

[49] M. Rudelson and R. Vershynin, Sparse reconstruction by convex relaxation: Fourier and Gaussian measurements, 40th Ann. Conf. Inf. Sc. syst. (2006).

[50] J. A. Tropp and A. C. Gilbert, Signal recovery from random measurements via orthogonal matching pursuit, IEEE Trans. Inf. Theory, 53(12) (2007) 4655-4666.

[51] D. Valsesia, and E. Magli, Compressive signal processing with Circulant sensing matrices, IEEE Int. Conf. Acoustics, Speech and Signal Processing, (2014) 1015-1019.

[52] W. Yin, S. P. Morgan, j. Yang, and Y. Zhang, Practical compressive sensing with Toeplitz and Circulant matrices, Rice University CAAM Technical Report TR10-01. (2014)1-6.

[53] H. Rauhut, J. Romberg, and J. A. Tropp, Restricted isometries for partial random Circulant matrices, Appl. Comput. Harmon. Anal., 32(2) (2012) 242-254.

[54] H. Rauhut, K. Schnass, and P. Vandergheynst, Compressed sensing and redundant dictionaries, IEEE Trans. Inf. Theory, 54(5) (2008) 2210-2219.

[55] T. L. Nguyen, and Y. Shin, Deterministic sensing matrices in compressive sensing: A survey, Sci. World J., (2013) 1- 6.

[56] D. L. Donoho, Y. Tsaig, I. Drori, J. Starck, Sparse solution of underdetermined systems of linear Equations by stagewise orthogonal matching pursuit, IEEE Trans. Inf. Theory 58(2) (2012) 1094-112.

[57] D. Needell, J.A. Tropp, CoSaMP: Iterative signal recovery from incomplete and inaccurate samples, Applied and Computational Harmonic Analysis, Elsevier, 26(3) (2009) 301-321.

[58] Z. Jellali, L. N. Atallah and S. Cherif, Greedy Orthogonal Matching Pursuit for sparse target detection and counting in WSN, Conf. European. Signal Process. (2014) 2250-2254.

[59] J. Wang, S. Kwon, and K. Shim, Generalized orthogonal matching pursuit, IEEE Trans. Signal. Process. 60(12) (2012) 6202-6216.

[60] X. Li, S. Hong, Z. Han, and Z. Wu, Bayesian compressed sensing based dynamic joint spectrum sensing and primary user localization for dynamic spectrum access,IEEE Glob. Telecommun. Conf. (2011) 1-5.

[61] Y. Tsaig, and D. L. Donoho, Extensions of compressed sensing, Signal Process. ELSEVIER, 86(3) (2006) 549-571.

[62] J. a. Tropp and A. C. Gilbert, Signal recovery from random measurements via orthogonal matching pursuit, IEEE Trans. Inf. Theory, 53(12) (2007) 4655-4666.

[63] S. Narayanan, S. K. Sahoo, and A. Makur, Greedy Pursuits Assisted Basis Pursuit for Compressive Sensing, Conf. European Signal Process. (2015).

[64] T. Blumensath and M. E. Davies, Iterative hard thresholding for compressed sensing, Appl. Comput. Harmon. Anal. 27(3) (2009) 265274.

[65] T. Blumensath and M. Davies, Normalized iterative hard thresholding: Guaranteed stability and performance, IEEE J. Selected Topics Signal Process. 4(2) (2010) 298309.

[66] S. Foucart, Hard threshol pursuit: An algorithm for compressive sensing, SIAM J. Numerical Analysis 49(6) (2011) $2543-2563$.

[67] N. Deanna, J. Tropp, and R. Vershynin, Greedy signal recovery review, IEEE Conf. Signals Syst. Computers, (2008)

[68] N. Deanna and R. Vershynin. Greedy signal recovery and uncertainty principles, Electronic Imaging. Inter. Society for Optics and Photonics, (2008).

[69] W. Dai, and O. Milenkovic, Subspace pursuit for compressive sensing: Closing the gap between performance and complexity, ILLINOIS UNIV at URBANA-CHAMAPAIGN, (2008).

[70] S. Qaisar, R. M. Bilal, W. Iqbal, M. Naureen, and s. Lee, Compressive sensing: From theory to applications, A Survey, Comm. Netw. J., 15(5) (2013) 443-456.

[71] S. Ji, Y. Xue, and L. Carin, Bayesian compressive sensing, IEEE Trans. Signal Process. 56(6) (2008) $2346-2356$.

[72] H. Reyes, S. Subramaniam, and N. Kaabouch, A Bayesian network model of the bit error rate for cognitive radio networks, IEEE Wirel. Microwave Tech. Conf. (2015).

[73]S. G. Mallat, Z. Zhang, Matching pursuits with time-frequency dictionaries, IEEE Trans. Signal Process. 41(12) $3397-3415$. 
[74] M. a. Davenport, P. T. Boufounos, M. B. Wakin, and R. G. Baraniuk, Signal processing with compressive measurements, IEEE J. Sel. Top. Signal Process. 4(2) (2010) 445-460.

[75] J. A. Tropp, Random Filters for Compressive Sampling, Ann. Conf. Info. Sci. Sys, (2006) 216-217.

[76] J. A. Tropp, M. B. Wakin, M. F. Duarte, D. Baron and R. G. Baraniuk, Random Filters for Compressive Sampling and Reconstruction, IEEE Inter.Conf. Acoustics Speech and Signal Processing, 3 (2006) III.

[77] J. Romberg, Sensing by random convolution, IEEE Inter. Workshop Computational Advances in Multi-Sensor Adaptive Processing, (2007) 137-140.

[78] J. Romberg, Compressive sensing by random convolution. SIAM J. Imaging Sci., 2(4) (2009) 1098-1128.

[79]A. Arif, and J. Romberg, Compressive Multiplexers for Correlated Signals, IEEE Asilomar Conf. Signals, Systems and Computers, (2012) 963-967.

[80] M. Mishali, Y. C. Eldar, and A. J. Elron, Xampling: Signal acquisition and processing in union of subspaces, IEEE Trans. Signal Process. 59(10) (2011) 4719-4734.

[81] J. Tropp, J. N. Laska, M. F. Duarte, J. K. Romberg, and R. G. Baraniuk, Beyond Nyquist: Efficient sampling of sparse bandlimited signals, IEEE Trans. Inf. Theory 56(1) (2010) 520-544.

[82] T. Strohmer, Measure What Should be Measured: Progress and Challenges in Compressive Sensing, IEEE Signal Processing Letters, 19(12) (2012) 887 - 893.[83] Z. Zhang and B. D. Rao, Sparse signal recovery in the presence of correlated multiple measurement vectors, ICASSP, IEEE Int. Conf. Acoust. Speech Signal Proc., (2010) 3986-3989.

[84] L. Liu, Z. Han, Z. Wu, and L. Qian, Collaborative compressive sensing based dynamic spectrum sensing and mobile primary user localization in cognitive radio networks, IEEE Glob. Telecommun. Conf., vol., no., pp. 1-5, 2011.

[85] Y. Polo and Y. Wang, Compressive wide-band spectrum sensing, IEEE Int. Conf. Acoust. Speech Signal Process. (2009) 2337-2340.

[86] A. M. Elzanati, M. F. Abdelkader, K. G. Seddik, and A. M. Ghuniem, Adaptive spectrum hole detection using sequential compressive sensing, IEEE Wirel. Commun. Netw. Conf. (2014) 1081-1086.

[87] H. Sun, W.-Y. Chiu, and A. Nallanathan, Adaptive compressive spectrum sensing for wideband cognitive radios, IEEE Commun. Lett.16(11) (2012) 1812-1815.

[88] S. S. Alam, M. O. Mughal, L. Marcenaro, and C. S. Regazzoni, Computationally efficient compressive sensing in wideband cognitive radios, Int. Conf. Next Gener. Mob. Appl. Serv. Technol. (2013) 226-231.

[89] M. Basaran, S. Erkucuk, and H. A. Cirpan, Achievable performance of bayesian compressive sensing based spectrum sensing, IEEE Int. Conf. Conf. on Ultra-WideBand, (2014) 86-90.

[90] H. C. Chen, H. T. Kung, D. Vlah, D. Hague, M. Muccio, and B. Poland, Collaborative compressive spectrum sensing in a UAV environment, Proc. IEEE Mil. Commun. Conf. (2011) 142-148.

[91] X. C. X. Chen, L. Z. L. Zhao, and J. L. J. Li, A modified spectrum sensing method for wideband cognitive radio based on compressive sensing, Fourth Int. Conf. Commun. Netw. (2009) 1-5.

[92] A. M. Elzanati, M. F. Abdelkader, K. G. Seddik, and A. M. Ghuniem, Collaborative compressive spectrum sensing using kronecker sparsifying basis, IEEE Wirel. Commun. Netw. Conf., (2013) 2902-2907.

[93] A. Appaiah, A. Perincherry, A. S. Keskar, and V. Krishna, Spectrum sensing in cognitive radio based on compressive measurements, Int. Conf. on Emerging Trends in Communication, Control, Signal Processing, and Computing Applications, (2013) 1-6.

[94] Z. Tian, Y. Tafesse, and B. M. Sadler, Cyclic feature detection with sub-Nyquist sampling for wideband spectrum sensing, IEEE J. Sel. Signal Process., 6(1) (2012) 58-69.

[95] H. Sun, W. Y. Chiu, and A. Nallanathan, Adaptive compressive spectrum sensing for wideband cognitive radios, IEEE Commun. Lett., 16(11) (2012) 1812-1815.

[96] D. Ariananda and G. Leus, Compressive wideband power spectrum estimation, IEEE Trans. Signal Process., 60(9) (2012) 4775-4789. [97] Y. Wang, Z. Tian, and C. Feng, Collecting detection diversity and complexity gains in cooperative spectrum sensing, IEEE Trans. Wirel. Commun., 11(8) (2012) $2876-2883$.

[98] Z. Qin, Y. Gao, M. D. Plumbley, C. G. Parini, L. G. Cuthbert, Low-rank matrix completion based malicious user detection in cooperative spectrum sensing, IEEE Global Conf. on Signal and Information Processing, (2013) 1186-1189.

[99] Z. Qin, Y. Gao and C. Parini, Data-assisted Low complexity compressive spectrum sensing on real-time signals under sub-Nyquist rate, IEEE Trans. Wirel. Comm., (2015).

[100] Z. Qin, L. Wei, Y. Gao and C. G. Clive, Compressive spectrum sensing augmented by Geo-location database, Inter.Workshop on Smart Spectrum at IEEE Wirel. Comm. Netw. Conf. (2015).

[101] Z. Qin, Y. Gao, M. Plumbley and C. Clive, Wideband spectrum sensing on real-time signals at sub-Nyquist sampling rates in single and cooperative multiple nodes, IEEE Trans. Signal Processing, (2015).

[102] J. Meng, W. Yin, H. Li, E. Hossain, Z. Han, Collaborative spectrum sensing from sparse observations in cognitive radio networks, IEEE J. Selected Areas Comm., 29(2) (2012) 327-337.

[103] G. Pope, Compressive sensing: A summary of reconstruction algorithms, Matrix (2009).

[104] C. Studer, P. Kuppinger, G. Pope, and H. Bölcskei, Recovery of sparsely corrupted signals (2011) 1-15.

[105] Y. Kim, W. Guo, B. V. Gowreesunker, N. Sun, and A. H. Tewfik, Multi-channel sparse data conversion with a single analog-to-digital converter, IEE J. Emerging and Selected Topics in Circuits and Systems, 2(3) (2012) 470-481.

[106] Ye, X., Zhu, W. P., Zhang, A., and J. Yan, Sparse channel estimation of MIMO- OFDM systems with unconstrained smoothed $10-$ norm-regularized least squares compressed sensing. EURASIP J. Wirel. Comm. Networking, (2013) 1-13.

[107] J. Xu, and G. Choi, Compressive sensing and reception for MIMO-OFDM based cognitive radio, Computing, Networking and Comm., Inter.1 Conf. (2015) 884-888.

[108] J. W. Choi and B. Shim, Compressive sensing based pilot reduction technique for massive MIMO systems, IEEE Information Theory and Applications Workshop, (2015) 115-118.

[109] G. Taubock, F. Hlawatsch, D. Eiwen, and H. Rauhut, Compressive estimation of doubly selective channels in multicarrier systems: Leakage effects and sparsity-enhancing processing, IEEE J. Selected Topics in Signal Processing, 4(2) (2010) 255-271.

[110] E. Chen, and C. Chu, Channel estimation for NC-OFDM systems based on subspace pursuit algorithm, IEEE Inter. Conf. Signal Processing, (2012) 88-91.

[111] M. Jia, X. Liu, and X. Gu, Channel estimation algorithm based on compressive sensing for NC-OFDM systems in cognitive radio context, Inter. J. Advancements in Computing Tech., 5(1) (2013).[112] C.R. Berger, Z. Wang, J. Huang, and S. Zhou, Application of compressive sensing to sparse channel estimation, IEEE Commun. Magazine, 48(11) (2010) 164-174.

[113] S. K. Sharma, E. Lagunas, S. Chatzinotas,B. Ottersten, Application of Compressive Sensing in Cognitive Radio Communications: A Survey, IEEE Communications Surveys \& Tutorials, PP(99) (2016) 1-24.

[114] M. Mishali, and Y.C. Eldar, From theory to practice: Sub-Nyquist sampling of sparse wideband analog signals, IEEE J. Selected Topics in Signal Processing, 4(2) (2010) 375-391.

[115] M. E. Lopes, Estimating unknown sparsity in compressed sensing, arXiv preprint arXiv:1204.4227, (2012). 